\title{
"ANOMALOUS" ETA PRODUCTION IN TAU DECAY
}

A. Pich

CERN - Geneva

\section{A B S T R A C T}

The decay modes of the $\tau$ lepton involving $\eta$ particles in the final state are analyzed. Some of these channels are related to the non-Abelian chiral anoma$1 y$ of QCD. It is found that the $\tau^{-} \rightarrow \nu_{\tau} \pi^{-} \eta \pi^{\circ}$ mode dominates the inclusive production of $\eta$ mesons in $\tau$ decay. 
The measured inclusive one-prong branching fraction of the $\tau$ lepton, $(86.8 \pm 0.3) \%$, is higher than the sum of the measured exclusive one-charged particle branching ratios 1$),(70 \pm 2) \%$ (decays involving more than one neutral meson are not included in the sum). This apparent discrepancy has motivated an increasing interest in the study of decay modes with multiple neutral mesons in the final state:

The experimental results reported during the last few months indicate that these channels can in fact account for the previously unidentified branching fraction, $\left(h^{\circ}=\pi^{\circ}, \eta\right)$

$$
\begin{aligned}
\operatorname{Br}\left(\tau^{-} \rightarrow v_{z} \pi^{-} \pi^{0}+n h^{0}, n \geqslant 1\right)= & (12.0 \pm 1.4 \pm 2.5) \%\left(\operatorname{MARK~II}{ }^{2)}\right) \\
& (13.9 \pm 2.0 \pm 1.9) \%\left(\mathrm{TPC}^{3)}\right)
\end{aligned}
$$

However, the available data also seem to show that a sizeable part of this rate cannot come from the expected dominant pion modes.

For the three-pion final state, a branching ratio of about $6 \%$ has been measured,

$$
\begin{aligned}
\operatorname{Br}\left(z^{-} \rightarrow v_{z} \pi^{-} \pi^{0} \pi^{0}\right)= & (6.0 \pm 3.0 \pm 1.8) \% & \left(\mathrm{CELLO}^{4}\right) \\
& (6.2 \pm 0.6 \pm 1.2) \% & \left(\text { MARK II }{ }^{5)}\right)
\end{aligned}
$$

which is consistent $\left.{ }^{*}\right)$ with the branching fraction for the $\tau^{-} \rightarrow \nu_{\tau} \pi^{-} \pi^{+} \pi^{-}$mode obtained by ARGUS ${ }^{6)},(5.6 \pm 0.7) \%, \operatorname{DELCO}^{7)},(5.0 \pm 1.0) \%, \operatorname{MAC}^{8)},(7.8 \pm 0.8) \%$ and MARK II $\left.{ }^{9}\right),(7.8 \pm 0.9) \%$. These results are in agreement with theoretical estimates 10$)$.

Using the $\mathrm{e}^{+} \mathrm{e}^{-} \rightarrow 2 \pi^{+} 2 \pi^{-}$data, Gilman and Rhie ${ }^{11)}$ predicted a $1 \%$ branching ratio for the $\tau^{-} \rightarrow \nu_{\tau} \pi^{-} 3 \pi^{\circ}$ decay. The experimental information on this mode is still quite uncertain,

*) From chiral symmetry one expects $B_{r}\left(\tau^{-} \rightarrow \nu_{\tau} \pi^{-} \pi^{0} \pi^{0}\right) \approx B_{r}\left(\tau^{-} \rightarrow \nu_{\tau} \pi^{-} \pi^{+} \pi^{-}\right)$. 


$$
\begin{aligned}
& \left.\operatorname{Br}\left(\tau^{-} \rightarrow v_{\tau} \pi^{-} \pi^{0} \pi^{0} \pi^{\circ}\right)=(3.0 \pm 2.2 \pm 1.5) \% \quad \text { (CELLO }{ }^{4)}\right) \\
& \left(0.0 \div+.4 \pm\left(\begin{array}{c}
1.1 \\
0.0
\end{array}\right) \% \quad \text { (MARK II }{ }^{5)}\right. \text { ) }
\end{aligned}
$$

In the CELLO analysis, modes with $\eta$ particles were not considered, while the MARK II result is obtained by allowing the $\tau^{-} \rightarrow \nu_{\tau} \eta \pi^{-} \pi^{0}$ channel to be present in the fit to the observed photon multiplicity spectrum, which then gives the surprisingly large result

$$
B r\left(\tau^{-} \rightarrow \nu_{2} \eta \pi^{*} \pi^{0}\right)=(4.2 \pm 1.1 \pm 1.6) \% \quad \text { (MARK II }{ }^{5)} \text { ) }
$$

If modes containing $\eta^{\prime}$ s are assumed to be absent, the same MARK II data ${ }^{5)}$ give a (2.2 \pm 0.4$) \%$ branching ratio for the $\tau^{-} \rightarrow \nu \tau^{-} 3 \pi^{\circ}$ decay, but the fit is then worse.

The Crystal Ball collaboration ${ }^{12), 13)}$ has also reported evidence for the $\tau \rightarrow \nu_{\tau} \eta \mathrm{X}$ channel which could imply a somewhat large (a few per cent) $\tau \rightarrow \nu_{\tau} \pi^{-} \pi^{\circ} \eta$ branching ratio. However, the analysis is still preliminary and awaits further background studies and efficiency calculations.

Decay modes with $\eta$ particles in the final state seem then to be more important than previously expected. Moreover, evidence for the second-class current channel $\tau^{+} \rightarrow \nu_{\tau} \pi^{+} \eta$ has been announced, with a branching ratio comparable to the one of typical first-class decays

$$
B_{r}\left(\tau^{+} \rightarrow \bar{z}_{\tau} \eta \pi^{+}\right)=(5.1 \pm 1.0 \pm 1.2) \% \quad\left(\mathrm{HRS}^{14}\right)
$$

These results must still be taken with some care in view of the difficulties inherent to this kind of experiment where a large number of photons are produced. In fact, while it is claimed ${ }^{14}$ ) that the HRS data on inclusive production of $\eta$ mesons in $\tau$ decay is not consistent with a final $\nu_{\tau} \eta \pi^{+} \pi^{\circ}$ state, the other experiments do not see the exotic $\nu_{\tau} \eta \pi^{+}$mode. However, bearing in mind the possibility of improved measurements which would clarify the situation, it is worthwhile analyzing what can be expected theoretically for these channels. 
At low momentum transfer, the coupling of a state $X$ of $n$ pseudoscalar mesons to the $\mathrm{V}-\mathrm{A}$ current $\mathrm{can}$ be estimated in a very easy way by using the effective chiral realization of QCD, which, to lowest order in derivatives and masses, is given by $\left(f_{\pi}=93.3 \mathrm{MeV}\right)$

$$
\mathscr{Z}_{\text {stiong }} \simeq \frac{f_{n}^{2}}{4} T_{r}\left(\partial_{\mu} u \partial^{\mu} u^{+}\right)+v T_{n}\left(M U+u^{+} M\right)
$$

The $3 \times 3$ special unitary matrix $U \equiv \exp \left(i \vec{\lambda} \vec{\phi} / f_{\pi}\right)$. incorporates the octet $\left.{ }^{*}\right)$ of pseudoscalar mesons, which appear as Goldstone co-ordinate fields $\vec{\phi}(x)$; M denotes the diagonal quark mass matrix, $M=\operatorname{diag}\left(m_{u}, m_{d}, m_{s}\right)$, and

$$
v=\frac{f_{\pi}^{2} m_{\pi^{*}}^{2}}{2\left(m_{u}+m_{d}\right)}=\frac{f_{\pi}^{2} m_{k^{*}}^{2}}{2\left(m_{n}+m_{s}\right)}=\frac{f_{\pi}^{2} m_{k^{0}}^{2}}{2\left(m_{d}+m_{s}\right)}
$$

In this realization, the left current is given by $(\Phi \equiv \vec{\lambda} \phi / \sqrt{ } 2)$

$$
\begin{aligned}
L_{\mu} & =(V-A)_{\mu}=i f_{N}^{2} U \partial_{\mu} u^{+} \\
& =\sqrt{2} f_{N} \partial_{\mu} \Phi+i\left(\Phi \vec{\partial}_{\mu} \Phi\right)-\frac{\sqrt{2}}{3 f_{N}}\left[\Phi,\left(\Phi \vec{\partial}_{\mu} \Phi\right)\right]+\ldots
\end{aligned}
$$

When the momentum transfer involved is large, the amplitudes

$$
T_{\mu}\left(P_{1}, \ldots, P_{n}\right) \equiv\left\langle x\left|L_{\mu} \exp \left\{i \int d^{*} z Z_{\text {strang }}(z)\right\}\right| 0\right\rangle
$$

obtained from Eqs. (1)-(3) must be continued from threshold by suitable final state interaction enhancements, which take into account the possible resonance structures present in each channel in a phenomenological way ${ }^{15)}$. This can be done by weighting the contribution of a given set of pseudoscalars, with definite quantum numbers, with the appropriate resonance form factor. The requirement that the chiral predictions must be recovered below the resonance region, fixes the normalization of these form factors to be one at zero invariant mass. I take the standard ansatz

$$
F_{R}(s)=\frac{M_{R}^{2}-i M_{R} \Gamma_{R}}{M_{R}^{2}-s-i M_{R} \Gamma_{R}}
$$

where $M_{R}\left(\Gamma_{R}\right)$ denote the mass (width) of the resonance $R$.

*) We neglect the $\eta-\eta^{\prime}$ mixing effects. 
It is we 11 known that the $\eta \pi^{-}$state, with quantum numbers $J^{p}=0^{+}, 1^{-}$and $I^{G}=I^{-}$, has a $G$ parity opposite to that of the vector current $V_{\mu}^{12}$, and therefore can only be produced in the standard model as an isospin violating effect. The decay width is then suppressed by the square of the u-d mass difference. The coupling to the vector current occurs through the $\eta-\pi^{\circ}$ mixing, which gives

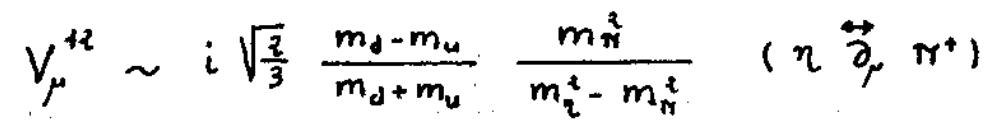

The $0^{+}$piece of this current is enhanced by the presence of the $a_{0}(980)$ resonance and therefore dominates the $\tau$ decay width into the $\eta \pi^{-}$channel. Using the known ${ }^{16)}$ mass $\left(M_{a_{0}}=983 \mathrm{MeV}\right)$ and width $\left(\Gamma_{a_{0}}=54 \mathrm{MeV}\right)$ of the $a_{0}(980)$ and the value $\left.{ }^{17}\right)\left(m_{d}-m_{u}\right) /\left(m_{d}+m_{u}\right) \simeq 0.28$ for the quark mass ratio, one finds a width of $7.1 \times 10^{-17} \mathrm{GeV}\left(2.8 \times 10^{-18} \mathrm{GeV}\right)$ for the $\tau^{-} \rightarrow v_{\tau} \pi^{-} \eta$ decay in the $0^{+}\left(1^{-}\right)$channel. This gives a total $\left(0^{+}+1^{-}\right)$branching ratio

$$
B_{r}\left(z^{-} \rightarrow \mu_{z} n^{-} \eta\right) \simeq 3.710^{-5}
$$

well below the HRS reported value.

For this mode, one can alternatively assume $a_{0}(980)$ dominance and use the $a_{0}$ decay amplitude, i.e., $T_{\mu}=\sqrt{ } 2 \mathrm{f}_{a_{0}} \mathrm{q}_{\mu}$ to evaluate the width with the result $\Gamma\left(\tau \rightarrow \nu_{\tau} a_{0}\right) \sim 3.1 \times 10^{-17} \mathrm{GeV} .\left(\mathrm{f}_{\mathrm{a}_{0}} / 1 \mathrm{MeV}\right)^{2}$. For the typical values of $\mathrm{f}_{\mathrm{a}_{0}}$ found in the literature $\left(1.5 \mathrm{MeV}^{18)}, 1.8 \mathrm{MeV}^{19)}, 1.3 \pm 0.2 \mathrm{MeV}^{20)}\right.$, this gives a similar branching ratio. In fact, using the chiral realization plus the $a_{0}$ enhancement of the divergence of the vector current, one can compute $f_{a_{0}}$ through the formula

$$
2 f_{a_{0}} \simeq \int d t \frac{1}{t^{2}} \frac{1}{\pi} \operatorname{Im} \psi(t)
$$

where $\phi(t)$ is the two-point function constructed with the divergence of the vector current $\partial^{\mu} v_{\mu}{ }^{21}$ ). In this way $I$ find $f_{a_{0}} \simeq 1.6 \mathrm{MeV}$, which agrees with the values given before. 
As the HRS experiment cannot discriminate between charged pions and kaons, its measurement is contaminated by the Cabibbo disfavoured (but normal, i.e., not suppressed by 1 ight quark mass differences) decay $\tau^{-} \rightarrow \nu_{\tau} \eta \mathrm{K}^{-}$. The relevant vector current is here

$$
V_{\mu}^{+3} \sim i \sqrt{\frac{3}{2}}\left(\eta \ddot{\partial_{\mu}} K^{+}\right)
$$

which gives the branching ratio

$$
B n\left(\tau^{-} \rightarrow \nu_{\tau} k^{-}\right) \simeq 1.210^{-4}
$$

Perhaps not so well known is the fact that the decay modes $\tau \rightarrow \nu_{\tau} \eta+\mathrm{n} \pi$ 's with $n>2$ are also "anomalous" ${ }^{22}$, but in a different sense. As the pion and the $\eta$ have a G-parity -1 and +1 , respectively, $G\left(\eta+n \pi^{\prime} s\right)=(-1)^{n}$ and the $\eta+n \pi^{\prime} s$ system with $n$ odd (even) can only couple, in the isospin conserved 1 imit, to the axial (vector) current. However, because of an accidental symmetry of the effective chiral Lagrangian, the axial (vector) current given in Eq. (3) only couples to an odd (even) number of pseudoscalars. Therefore, the decays $\tau \rightarrow \nu_{\tau} \eta+n \pi^{\prime}$ s break G-parity and should also be suppressed by the $\left(m_{d}-m_{u}\right)^{2}$ factor.

The above conclusion would be true if the chiral symmetries of the QCD Lagrangian were preserved by the quantized theory. However, we know that the natve Ward identities are affected by the non-Abelian chiral anomaly. In the low energy world, this implies the existence of an additional piece in the strong effective Lagrangian, the Wess-2umino term ${ }^{23), 24)}$, which in turn gives an additional contribution to the left current

$$
L_{\mu}^{(a n+)} \equiv(V-A)_{\mu}^{(a n .)}=-i \frac{N_{c}}{24 \pi^{2} f_{\pi}^{6}} \varepsilon_{\mu \omega \alpha \beta} L^{\omega} L^{\alpha} L^{\beta}
$$

The characteristic anomaly structure of this current (i.e., the presence of the Levi-Civita pseudotensor) just reverses the parity argument: $A_{\mu}^{(a n .)}\left(v_{\mu}^{(a n .)}\right)$ couples to an even (odd) number of pseudoscalars.

The decay modes $\tau \rightarrow \nu_{\tau} \eta+\mathfrak{n} \pi{ }^{\prime} s(n>2)$ are therefore allowed without any isospin suppression (note that this does not apply for $\tau^{-} \rightarrow \nu_{\tau} \eta \pi^{-}$because $\mathrm{L}_{\mu}$ (an.) 
only makes a contribution to systems with at least three mesons). Its mere detection at a non-suppressed level provides an experimental signal of the non-Abelian anomaly of QCD. Unfortunately, the presence of resonances at the high $Q^{2}$ values relevant for the $\tau^{-}$decay spoils the possibility of making a clean quantitative test of the anomaly predictions.

The "anomalous" channel with lowest meson multiplicity is the $\eta \pi^{-} \pi^{\circ}$ one, which is produced by the vector piece

$$
V_{\mu}^{(a n, 1+2} \simeq \frac{i \varepsilon_{\mu \omega \alpha \beta}}{4 \sqrt{6} \pi^{2} f_{\pi}^{3}} \quad \partial^{\alpha} \eta \quad \partial^{\alpha}\left(\pi^{+} \partial^{\beta} \pi^{0}\right)
$$

This mode is enhanced by the presence of the $\pi \pi \rho(770)$ and the $\eta \pi \pi \rho(1600)$ resonances $^{*}$ ). The three-derivative coupling tends to peak the invariant mass distribution in the high $Q^{2}$ region where the $\rho(1600)$ enhancement is present. This makes the numerical estimate of the rate very sensitive to the exact values of the resonance parameters. Taking the particle data group central values 16 ) $\left(M_{\rho}(1600) \sim 1.59 \mathrm{GeV}, \Gamma_{\rho(1600)} \sim 0.26 \mathrm{GeV}\right)$, one gets a $1 \%$ branching ratio for this decay ${ }^{* *}$. However, if the $\rho(1600)$ is a much broader resonance as suggested by the $\mathrm{e}^{+} \mathrm{e}^{-} \rightarrow 4 \pi \mathrm{data}^{25)}$, the decay rate is smaller. I take $\Gamma_{\rho(1600)} \sim 0.5 \mathrm{GeV}$, which is also favoured ${ }^{10)}$ by the $e^{+} e^{-} \rightarrow \eta \pi^{+} \pi^{-}$results ${ }^{26)}$. This gives

$$
B_{5}\left(\tau^{-} \rightarrow \omega_{2} \eta \pi^{-} \pi^{0}\right) \sim 0.3 \%
$$

*) A possible $\rho(1250)$ resonance in the $\eta \pi \pi$ channel is excluded by the $e^{+} e^{-} \rightarrow$ $\eta \pi^{+} \pi^{-}$measurements 26$\left.), 27\right)$.

**) This result disagrees with the estimate made in Ref. 22), which gives a very low branching ratio $\left(B_{r} \sim 4 \times 10^{-4}\right)$. In this calculation, an $\eta \pi \pi \rho(770)$ resonance which interferes destructively with the $\eta \pi \pi \rho(1600)$ one is considered. Moreover, by introducing an additional small coupling $g_{\rho^{\prime}} \pi \pi^{/ g} \rho^{\prime}$, the authors of Ref. 22) completely kill the $\rho(1600)$ contribution. As shown before with the $f_{a}$ estimate, the chiral parametrization already contains information about quantities like $\mathrm{f}_{a_{0}}$. Therefore, additional narrow width couplings should not be introduced. 
The next "anomalous" modes in order of meson multiplicity are the one-prong $\tau^{-} \rightarrow \nu_{\tau} \eta \pi^{-} \pi^{0} \pi^{0}$ and the three-prong $\tau^{-} \rightarrow \nu_{\tau} \eta \pi^{-} \pi^{+} \pi^{-}$, which are generated by the axial current

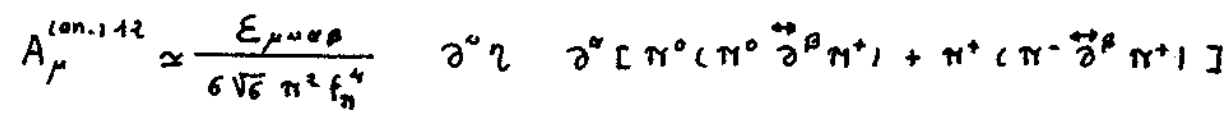

and therefore the width is predicted to be the same for both channels. The presence of the $\pi \pi \pi a_{1}(1270)$ resonance cannot overcome the big phase space suppression which amounts to a very small branching ratio

$$
B_{r}\left(z^{-} \rightarrow \omega_{\varepsilon} 2 \pi^{-} \pi^{0} \pi^{0}\right) \simeq B_{r}\left(2^{-} \rightarrow \nu_{z} \eta \pi^{-} \pi^{+} \pi^{-1} \simeq 1.210^{-6}\right.
$$

The non-"anomalous" mode $\eta \eta \pi^{-}$is also peculiar because, in the chiral limit, it is not generated by the axial current. This can be understood by noticing from Eq. (3) that the three-meson axial current is in fact constructed from the twomeson vector one, $\left(\dot{\Phi}_{\mu} \Phi\right)$, which obviously cannot give any $\eta \eta$ (antisymmetry) or $\eta \pi$ (G-parity) contribution. When masses are turned on, this decay occurs through the divergence of the axial current

$$
\partial^{\mu} A_{\mu}^{12} \simeq \frac{m_{\pi}^{2}}{3 \sqrt{2} f_{\pi}} \pi^{+} \eta^{2}
$$

but, of course, at a very low rate

$$
\operatorname{Br}\left(2^{-} \rightarrow \alpha_{2} \eta \eta r^{-}\right) \simeq 1.110^{-9}
$$

There are only four additional modes, with meson multiplicity three, which contain $\eta$ particles in the final state: $\eta \mathrm{K}^{\circ} \mathrm{K}^{-}, \eta \pi^{-} \overline{\mathrm{K}}^{\circ}, \eta \pi^{\circ} \mathrm{K}^{-}$and $\eta \eta \mathrm{K}^{-}$. As these channels contain kaons which have no definite G-parity quantum number, they get

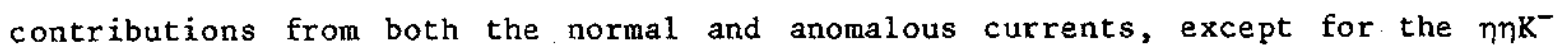
final state which does not couple to $v_{\mu}^{(a n .)}$ because the two etas cannot be in an antisymetric state.

The Cabibbo favoured $\eta \mathrm{K}^{\circ} \mathrm{K}^{-}$mode is very suppressed because of the small phase space available. It has a total branching ratio of 


$$
B_{r}\left(\tau^{-} \rightarrow \nu_{\tau} \eta k^{0} k^{-}\right) \simeq 3.110^{-7}
$$

About $44 \%$ of the $\eta \mathrm{K}^{\circ} \mathrm{K}^{-}$decay width corresponds to the anomaly contribution. The same phase space suppression completely kills the already Cabibbo disfavoured $\eta \eta \mathrm{K}^{-}$mode

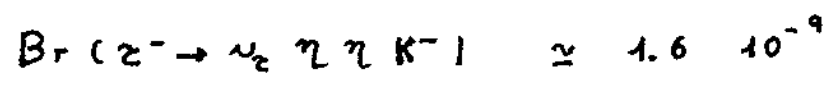

The remaining Cabibbo suppressed modes $\eta \pi^{-} \bar{K}^{\circ}$ and $\eta \pi^{\circ} K^{-}$, have a bigger phase space and, moreover, contain resonant $K^{*}$ contributions which enhance them slightly, giving the branching ratios

$$
\begin{aligned}
& B_{r}\left(2^{-} \rightarrow \omega_{2} \eta \pi^{-} \bar{K}^{0}\right) \simeq 2.210^{-5} \\
& \operatorname{Br}\left(2^{-} \rightarrow \omega_{\tau} \eta \pi^{0} k^{-}\right) \simeq 8.810^{-6}
\end{aligned}
$$

In conclusion, the inclusive production of $\eta$ mesons in the $\tau$ decay is dominated by the purely "anomalous" channel $\tau^{-} \rightarrow \nu_{\tau} \eta \pi^{-} \pi^{\circ}$. This provides a nice qualitative signal of the non-Abelian chiral anomaly of QCD. The quantitative estimate of this decay mode is quite uncertain because of the not very well known $\eta \pi \pi \rho(1600)$ resonance. Although a branching ratio as 1 arge as $1 \%$ can be obtained by using a "narrow" $\rho(1600)$ width of $\Gamma_{\rho(1600)} \sim 0.26 \mathrm{GeV}$, a more moderate result of about $0.3 \%$ is obtained for a broader $\rho(1600)\left(\Gamma_{\rho}(1600) \sim 0.5 \mathrm{GeV}\right)$, as suggested by the $\mathrm{e}^{+} \mathrm{e}^{-} \rightarrow 4 \pi$ and $\mathrm{e}^{+} \mathrm{e}^{-} \rightarrow \eta \pi \pi$ measurements.

Note added

An estimate of the $\tau^{-} \rightarrow \nu_{\tau} \eta \pi^{-} \pi^{\circ}$ decay rate has recently been done ${ }^{28)}$ by relating $i t$, through an isospin rotation, to the experimental data on $\mathrm{e}^{+} \mathrm{e}^{-} \rightarrow n \pi^{+} \pi^{-}$. In this way a branching ratio of about $0.2 \%$ has been found. I thank $\mathrm{J}$. Ellis for making me aware of this reference.

\section{Acknowledgements}

I am indebted to $E$. de Rafael for useful suggestions and coments. I also wish to acknowledge discussions with J. Ellis and S. Narison. This work has been supported in part by CAICYT (Spain) under grant $\mathrm{Nr}$. 1740/82. 


\section{REFERENCES}

1) P.R. Burchat - Santa Cruz Institute for Particle Physics Report Nr. 86/72 (1986), to be published in Proceedings of the 23rd International Conference on High Energy Physics, Berkeley, 1986.

2) P.R. Burchat et al. - SLAC-PUB-4006 and LBL-21915 (June 1986).

3) H. Aihara et al. - Phys.Rev.Lett. 57 (1986) 1836.

4) H.J. Behrend et al. - Z.Phys. C23 (1984) 103.

5) K.K. Gan et a1. - SLAC-PUB-4110 and LBL-22362 (February 1987).

6) H. Albrecht et a1. - Z.Phys. C33 (1986) 7.

7) W. Ruckstuhl et a1. - Phys.Rev.Lett. 56 (1986) 2132.

8) Communication to the 23rd International Conference on High Energy Physics, Berkeley, 1986. Quoted in Ref. 1).

9) W.B. Schmidke et al. - Phys.Rev.Lett. 57 (1986) 527.

10) A. Pich - in preparation.

11) F.J. Gilman and S.H. Rhie - Phys.Rev. D31 (1985) 1066.

12) S.T. Lowe - SLAC-PUB-4151 (December 1986).

13) S. Keh - Communication to the 23rd International Conference on High Energy Physics, Bexkeley, 1986. Quoted in Ref. 1).

14) M. Derrick et a1. - ANL-HEP-PR-86-106, IUHEE-102, UM-HE-86-28 and PU-86-586 (February 1987).

15) R. Fischer, J. Wess and F. Wagner - 2.Phys. C3 (1980) 313.

16) M. Aguilar-Benitez et al. - "Review of Particle Properties", Phys.Lett. 170B (1986).

17) J. Gasser and H. Leutwyler - Physics Reports 87C (1982) 77.

18) S. Tisserant and T.N. Truong - Phys.Lett. 115B (1982) 264.

19) S. Narison, N. Paver, E. de Rafael and D. Treleani - Nuc1.Phys. B212 (1983) 365.

20) S. Narison - Proceedings of the 3rd Lear Workshop "Physics with Antiprotons at Lear in the Acol Era" (Tignes 1985), eds. U. Gastaldi et al., Editions Frontieres (1985), p. 297.

21) C.A. Dominguez and E. de Rafael - Ann.Phys. 174 (1987) 372.

22) G. Kramer and W.F. Palmer - Z.Phys. C25 (1984) 195. 
23) J. Wess and B. Zumino - Phys.Lett. 37B (1971) 95.

24) E. Witten - Nuc1.Phys. B223 (1983) 422.

25) A. Cordier et al. - Phys.Lett. 109B (1982) 129,.

26) B. Delcourt et a1. - Phys.Lett. 113B (1982) 93.

27) V.P. Drughinin et a1. - Phys.Lett. B174 (1986) 115.

28) F.J. Gilman - SLAC-PUB-4265 (March 1987). 